\title{
KATHARINA SIMON-MUSCHEID
}

\section{Les rois des compagnons de métiers}

En 1412, à Zurich, le roi des compagnons forgerons confirme par son sceau, pour sa personne et pour tous ses successeurs, au nom de tous les compagnons de son métier, un document important pour ce métier (fig. 1 et 2):

Ze urkúnd han ich obgen(ant) kúng min insigel von miner und aller schmidtknechten wegen, die dis vorgenant sach anlanget, offenlich und wissentlich gehenket an disen brief, der geben ist an der heiligen kindlinen tag nach Wienacht nach gotz geburt vierzehenhundert iar und in dem drizehenden jar ${ }^{1}$.

Le sceau est aujourd'hui conservé aux archives de la ville de Zurich. Il nous permet de retracer l'histoire d'une institution méconnue pour une vaste région située autour de l'Alsace, la Forêt-Noire, le lac de Constance et le nord-est de la Suisse: les rois des compagnons de métiers.

Au bas Moyen Âge, les compagnons de métiers de ces régions conclurent des accords qui dépassaient le cadre de leur ville. En formant des alliances interurbaines et interrégionales, ils adoptèrent le modèle préféré de leurs contemporains. Pour mieux défendre leurs intérêts communs et pour mieux se protéger, toutes les couches sociales avaient commencé à s'allier de cette manière, des chevaliers (Ritterbünde) et des villes (Städtebünde), à la population mobile, jusqu'aux groupes situés plus en marge de la société (comme les ménétriers alsaciens) ${ }^{2}$. Les maîtres artisans et les compagnons suivirent leur exemple. Comme eux, les compagnons utilisaient leurs réseaux étendus et des voies de communication bien établies ${ }^{3}$. Organisées en royaumes, ces associa-

1 Cité par Friedrich HEgI, Geschichte der Zunft zu Schmieden in Zürich 1336-1912, Zurich 1912, p. 129. Le document et le sceau se trouvent aux archives de la ville de Zurich, archives de la corporation des forgerons, doc. 3. Le texte est également publié dans: Werner SCHNYDER, Quellen zur Zürcher Zunftgeschichte, t. 1: 13. Jahrhundert bis 1604, Zurich 1936, p. 68, n. 58.

2 Sur ce phénomène typique du bas Moyen Âge, voir František GRAUS, Organisationsformen der Randständigen. Das sogenannte Königreich der Bettler, dans: Rechtshistorisches Journal 8 (1989), p. 235-255.

3 Lucien SitTLER, Les associations artisanales en Alsace au Moyen Âge et sous l'Ancien Régime, dans: Revue d'Alsace 97 (1958), p. 36-80. À part les associations des ménétriers (Pfeifer), des chaudronniers et des potiers existaient au Moyen Âge, plus ou moins étendues, des associations de tuiliers, barbiers, tailleurs de pierre, cordonniers (comprenant entre autres des territoires habsbourgeois de la Haute-Alsace et du Brisgau, les villes de Bâle, Mulhouse, Colmar, Sélestat, Kaysersberg, Fribourg, Brisach), selliers (constituée en 1435 à Schaffhouse et comprenant des villes de Souabe, de Franconie et de Suisse), tisserands, meuniers, et boulangers, avec parmi elles des associations mixtes comprenant maîtres et compagnons, ibid., p. 37-69. D’après Frank GöTtMANN, Handwerk und Bündnispolitik. Die Handwerkerbünde am Mittelrhein vom 14. bis zum 17. Jahrhundert, Wiesbaden 1977, p. 10, la forêt de Haguenau et la ville de Coblenz marquaient les limites entre la vallée du haut Rhin et celle du haut Rhin moyen. Voir 
tions furent dirigées par des rois. Ces rois étaient élus par les membres eux-mêmes et confirmés par leur seigneur ou bien encore imposés par lui ${ }^{4}$.

Mais les deux associations que j'aimerais vous présenter, celle des compagnons forgerons et celle des cordonniers, se distinguent nettement des autres royaumes rencontrés: elles ont été créées par les compagnons eux-mêmes et imposées aux maîtres des corporations par la suite. Les compagnons, durant le premier quart du $\mathrm{XV}^{\mathrm{e}}$ siècle, étaient devenus des groupes puissants et conscients de leur valeur sur le marché du travail ${ }^{5}$. Hormis pour ces deux groupes, il ne semble pas y avoir eu d'autres rois de compagnons (à ma connaissance). En revanche, d'autres métiers avaient essayé d'imiter ces structures, mais leurs efforts avaient échoué, soit par l'intervention d'autorités urbaines, soit par le trop petit nombre des compagnons regroupés. Les compagnons tailleurs, cordiers et pelletiers, probablement en train de se regrouper en associations similaires, avaient aussi possédé des sceaux, mais qui furent cassés par les autorités ${ }^{6}$.

Quant aux structures, les sources, rares et imprécises, proviennent surtout de la correspondance entre les villes ainsi que de certaines ordonnances. En 1408, par exemple, les membres du conseil de ville de Kaysersberg informent leurs collègues strasbourgeois des méfaits commis par un compagnon cordonnier, tout en précisant que les fonctionnaires élus par les compagnons cordonniers et tailleurs nuisent à la corporation. Pour désigner les dirigeants, ils emploient les termes de Schultheiss, Heger, Amtleute:

aussi Anne-Marie DuBLER, Handwerk, Gewerbe und Zunft in Stadt und Landschaft Luzern, Lucerne, Stuttgart 1982, p. 83-107.

4 GRAUS, Organisationsformen (voir n. 2), p. 252-253, confirme que le phénomène des rois et des royaumes, sous-estimé en Allemagne, était pourtant répandu. Notamment, il évoque des exemples alsaciens et suisses, à Genève le rex archeriorum, nommé lors des fêtes de tir, ou la reine des prostituées, qui tous deux exerçaient des fonctions essentiellement de contrôle. Il résume que les associations des marginaux institutionnalisées et contrôlées par les autorités n'exclurent point la participation active des marginaux. Selon Wolfgang SEIDENSPINNER, Das »Königreich« als Organisationsform gesellschaftlicher Gruppen. Soziale Integration, Geselligkeit, Alternative und Rebellion (vornehmlich nach oberrheinischen Quellen des Spätmittelalters), dans: Zeitschrift für die Geschichte des Oberrheins 146 (1998), p. 249-270, ces royaumes bien structurés et contrôlés par les seigneurs ou les autorités de villes offrirent une participation sociale aux groupes mobiles.

5 Voir Knut SCHULZ, Handwerksgesellen und Lohnarbeiter. Untersuchungen zur oberrheinischen und oberdeutschen Stadtgeschichte des 14. bis 17. Jahrhunderts, Sigmaringen 1985, p. 61-81; Kurt WESOLY, Lehrlinge und Handwerksgesellen am Mittelrhein. Ihre soziale Lage und ihre Organisation vom 14. bis 17. Jahrhundert, Francfort/M. 1985, p. 349-358; Wilfried REININGHAUS, Die Entstehung der Gesellengilden im Spätmittelalter, Wiesbaden 1981, p. 49188; Jean-Robert ZIMMERMANN, Les compagnons de métiers à Strasbourg du début du XIV ${ }^{\mathrm{e}}$ siècle à la veille de la Réforme, Strasbourg 1971, p. 99-118; Georg ScHANZ, Zur Geschichte der deutschen Gesellenverbände, Leipzig 1877; Franz J. MONE, Gewerbepolizei vom 12. bis 18. Jahrhundert in der Schweiz, Baden, Württemberg, Zollern, Elsass und Hessen, dans: Zeitschrift für die Geschichte des Oberrheins 13 (1861), p. 129-163, 273-317.

6 En 1421, le conseil de ville de Bâle casse les sceaux des compagnons cordiers et pelletiers bâlois, voir REININGHAUS, Die Entstehung (voir n. 5), p. 28. Celui des compagnons tailleurs bâlois avait été cassé en 1389 par les autorités qui, en plus, interdirent leur association et bannirent leurs chefs. On peut supposer qu'ils étaient en train d'établir là une alliance interurbaine de Zurich à Constance, voir SCHULZ, Handwerksgesellen (voir n. 5), p. 76. 

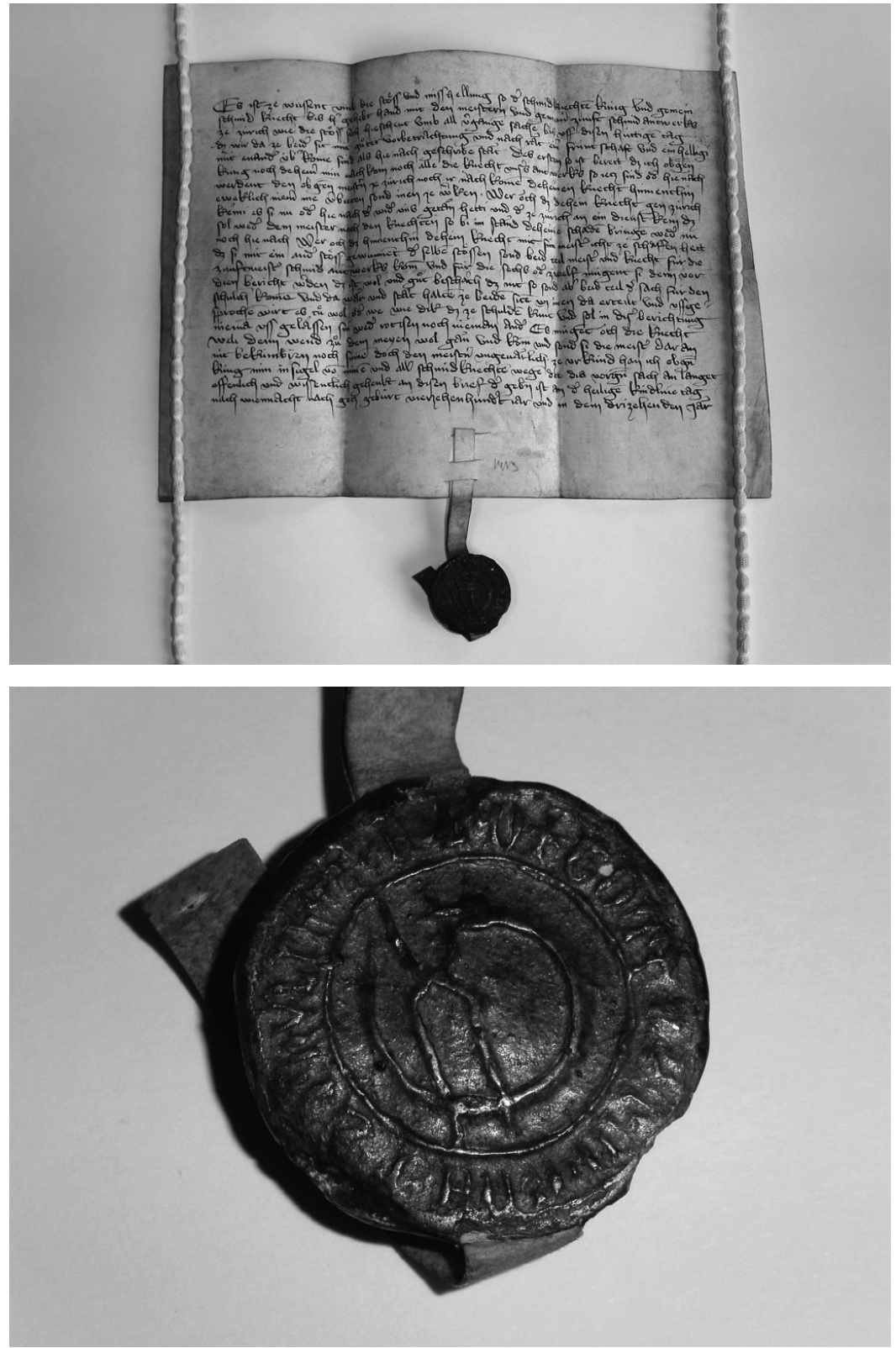

Fig. 1 und 2 (détail): Corporation des forgerons. Diplôme du 28 décembre 1412. Archives de la ville de Zurich, VII.179:1.3. Cliché: archives de la ville de Zurich. 
Lieben fründe. alz hant unsre erbern botten vor ziten wol fürbracht, wie ir und andere der richestetten botten unsre fründe mit einander geret hant von der antwerkknechte wegen besunder schumacher- und sniderknecht, wie die gerichte under inen selber haltent und schultheissen und heger und amplüte under inen satzent und den gehorsam sint, denne den herscheften und meistern und reten, under den sü denne wonende sint, daz doch wider daz rich ist und richen und armen in dem lande wonende grossen schaden bringet. nun hant ir derselben knechte einen by uoch wonende als uns fürkommen ist, heisset Rosenzwig, der het einem meister by uns wonende schuohemacherantwerkes, heisset meister Snewelin, knehte verbotten, alz im daz ander knehte sins antwerkes kunt geton hant, uober daz das er doch nuot mit im ze tuonde hat denne guotes alz er sprichet, und leit im sine werkstat muossig und bringet in zuo grossem verderplichen schaden und bringet in darzuo daz er von dem riche wichen muos ${ }^{7}$.

On peut supposer qu'au début du $\mathrm{XV}^{\mathrm{e}}$ siècle, les compagnons cordiers, à leur tour, cherchèrent à s'organiser à la manière des forgerons. La ville de Mayence, ayant appris que les cordiers avaient l'intention de former une alliance interurbaine (ou le craignant), leur interdit formellement d'élire un roi sans en avoir obtenu l'autorisation par les autorités urbaines ${ }^{8}$. La structure de leur association, très active jusqu'au milieu du $\mathrm{XV}^{\mathrm{e}}$ siècle demeure cependant inconnue, et le titre de »roi« n'apparaît pas dans les documents 9 .

\section{LES MODÈLES \\ LES MÉNÉTRIERS ET LES CHAUDRONNIERS}

Le royaume le plus connu pour notre région est sans nul doute celui des ménétriers d'Alsace. Cette royauté était un fief impérial tenu par les seigneurs de Ribeaupierre, qui déléguèrent les fonctions de cette royauté aux ménétriers eux-mêmes. En 1400, le seigneur Smassmann de Ribeaupierre nomma un certain Henselin, fifre, roi de tous les ménétriers d'Alsace. Ce royaume »des gens ambulants« (das kúnigrich farender lúte) fut délimité explicitement par la forêt de Haguenau, la Birse (un fleuve qui traverse le nord-est de la Suisse), le Rhin et les montagnes des Vosges:

Das kúnigrich farender lúte zwúschent Hagnoewer vorste vnd der Byrse, dem Ryne vnd der Virst $[\ldots]$ und lihe es ouch mit disem mime offenen briefe [...] mime pfiffer und varenden manne, also daz er das selbe kúnigrich vnd ambacht fúrbasser me sol haben, besiczzen, nvtzzen vund nies$\operatorname{sen}^{10}$.

7 Schanz, Zur Geschichte (voir n. 5), p. 184. Nous avons cité la partie de la lettre qui décrit les effets du boycottage; voir aussi SCHULZ, Handwerksgesellen (voir n. 5), p. 75.

8 Ernst SchUBerT, Fahrendes Volk im Mittelalter, Bielefeld 1995, p. 132, n. 3: keinen konig unter ihne machen in dutschen landen dan mit willen eines waltpoten (cité d'après Heinrich GRIMM, Weisthümer, vol. 1, Göttingen 1840, p. 533).

9 En 1451, lors d'une assemblée mixte des maîtres et des compagnons tenue à Bâle, les autorités, provoquées par l'arrogance des compagnons, les firent tous arrêter. Ils confisquèrent les lettres (malheureusement non spécifiées) trouvées chez leurs prisonniers, cassèrent leur sceau et interdirent leur association, voir SCHULZ, Handwerksgesellen (voir n. 5), p. 79-80.

10 Karl AlBrecht (éd.), Rappoltsteinisches Urkundenbuch 759-1500, t. 2, Colmar 1891-1898, p. 497, n. 643 (20 avril 1420). 
Le vice-roi, nommé par le seigneur de Ribeaupierre, et un comité de douze personnes (Zwölfer) formaient un tribunal (Pfeifergericht) ${ }^{11}$. Les règlements, dont les plus anciens datent de la fin du $\mathrm{XV}^{\mathrm{e}}$ siècle, reflètent d'une part la structure féodale de ce royaume et d'autre part son aspect confraternel. Les ménétriers rendent hommage au seigneur de Ribeaupierre, qui, à son tour, garantit de les protéger ${ }^{12}$. Quant à l'assemblée annuelle tenue à Ribeauvillé (Rappoltsweiler) le jour de la Nativité de la Vierge, elle commence par un cortège, suivi par l'office religieux célébré en l'église des Augustins et par la séance du tribunal ${ }^{13}$. Comme dans les confréries, l'assemblée des ménétriers (Pfeifertag) donnait lieu à une fête ${ }^{14}$.

Des royaumes de ménétriers existaient également en Suisse. À Zurich, en 1430, un »roi des fifres« est confirmé par les autorités de la ville après avoir été élu par les gens ambulants de toute la Confédération suisse:

[Ulman Meyers von Bremgarten, des Pfeifers] ernstlich bett [um Belehnung] haben wir angesechen, und sunder betrachtet, dass er von anderen varenden lüthen in der Eidtgnossenschaft einmüttenlich erwelt ist, und haben im auch das obgenannt pfiffer künigrich in allen unsseren graffschaften, herschafften, gerichten, gebieten, zwingen und bännen güttlich und gnedeklich verlichen ${ }^{15}$.

De la même manière, à Berne, en 1507, l'élection d'un roi de la confrérie des ménétriers est confirmée par un document scellé par le sceau du roi des confrères et signé de la manière suivante: Ich, Hans Ganter, der spillúten kung in miner heren stat und land zu Beren und ich, Hans Steinhoffer, sin stathalter ${ }^{16}$.

11 Sur les tribunaux, voir GRAUS, Organisationsformen (voir n. 2).

12 Chaque musicien doit comme impôt annuel à son seigneur deux sacs d'avoine et une poule, voir SCHUBERT, Fahrendes Volk (voir n. 8), p. 134; SEIDENSPINNER, Das »Königreich« (voir n. 4), p. 253-259; Martin VOGELEIS, Quellen und Bausteine zu einer Geschichte der Musik und des Theaters im Elsass 500-1800, Strasbourg 1911, p. 99. AlBRECHT (éd.), Rappoltsteinisches Urkundenbuch (voir n. 10), vol. 3, p. 386, n. 808 (10 avril 1431) et 809 (1434): le seigneur de Ribeaupierre donne ordre au roi des fifres de collecter l'avoine due. Voir aussi Maria DOBOZY, Re-membering the Present. The Medieval German Poet-ministrel in Cultural Context, Turnhout 2005.

13 D'après SitTLER, Les associations (voir n. 3), p. 37-38, le royaume comprenait au XV siècle cinq subdivisions: Vieux-Thann, Ribeaupierre, Sélestat, Rosheim et Strasbourg.

14 Ceux qui manquaient l'assemblée étaient sévèrement punis, ceux qui s'excusaient payaient le repas en plus de la cotisation, voir ibid. p. 38. Le Pfeifertag existait jusqu'à la Révolution. Pour la description d'un Pfeifertag au XVIII ${ }^{\mathrm{e}}$ siècle, voir VoGELEIS, Quellen (voir n. 12), p. 434436. Le repas avait lieu avant la tenue du tribunal et l'accueil des nouveaux membres. Le même programme se retrouve à peu près pour les assemblées des confréries de miséreux, aveugles et mendiants, voir Katharina SIMON-MUSCHEID, La fête des mendiants. Fictions et réalités au bas Moyen Âge (Bâle et Cologne), dans: Marc BoONE, Peter STABEL (dir.), Shaping Urban Identity in Late Medieval Europe, Leuven, Apeldoorn 2000, p. 183-200.

15 Le document est publié par M. OTT, dans: Anzeiger für Schweizerische Geschichte und Altertumskunde 1 (1856), p. 28.

16 Le document est publié par Heinrich THÜRLER, dans: Anzeiger für Schweizerische Geschichte und Altertumskunde 29 (1898), p. 17-18. 
L'autre exemple d'organisation qui pouvait servir de modèle aux compagnons de métiers était celui des chaudronniers ${ }^{17}$. Leurs membres, dispersés en petit nombre dans les villes et les villages, avaient formé une vaste association aussi ancienne que celle des ménétriers. Comme les corporations, ils défendaient leur monopole contre les concurrents libres et étrangers. Étendue dans tout l'Empire, leur association était divisée en cercles (Kesslerkreise) dont chacun avait à sa tête un seigneur comme roi et protecteur. Depuis 1361, les seigneurs alsaciens de Ratsamhausen avaient hérité de cette fonction au titre de leur fief d'Empire, fonction qu'ils détinrent jusqu'à la Révolution. En 1434, leur cercle fut délimité géographiquement par la forêt de Haguenau, le Hauenstein (sommet jurassien au nord-ouest de la Suisse), les crêtes des Vosges et celles de la Forêt-Noire. Comparable au royaume des ménétriers, ce cercle comprenait l'Alsace, le nord-ouest de la Suisse et le pays de Bade. La structure de cette organisation ressemblait à celle des ménétriers; les chaudronniers prêtaient serment de fidélité à leur seigneur et étaient obligés de garder son château de Ratsamhausen, de se mettre à son service un mois par an (quinze jours à leurs propres frais et quinze jours aux frais du seigneur) et de lui fournir la vaisselle en cuivre dont il avait besoin. Le seigneur, de son côté, les protégeait et défendait leurs intérêts ${ }^{18}$. Comme le seigneur de Ribeaupierre, il avait, dès 1434, délégué son autorité à un Schultheiss (écoutète ou prévôt), qui présidait les assemblées annuelles tenues dans la ville de Brisach, située au milieu du cercle.

Trois cercles s'étendaient sur le territoire de la Confédération suisse. Au nord-ouest les cercles de Ratsamhausen et de Königsegg, à l'est le cercle de Uechtland/Bourgogne et à l'ouest celui de Choir. Pendant les années 1420 et 1430, trois nouveaux cercles, plus petits que les anciens fiefs d'Empire, furent créés sur le territoire suisse: les trois royaumes de Berne, de Lucerne et de Zurich $^{19}$. Les statuts des chaudronniers bernois nous informent sur la position de leur roi. Ce document, dont nous avons choisi de citer les parties les plus intéressantes, est une étrange combinaison d'ordonnances de métier, de statuts de confrérie et d'obligations entre seigneur et vassaux:

[1] Des ersten sol das hantwerck mit unserm wússent und willen einen kúng setzen.

[2] Item hand si gemacht und geordnet, das ir kúng mit irem raet inen einen schulth[esse]n, der des handwercks sy, geben mag, welen er wil. Der selb schulthes sol ouch inen sweren zuo gott und den heiligen trúw und waerheit zeleisten, gewaertig, gehorsam und hilfflich zesinde, dartzuo si recht habent und mit guoter alter herkomenheit harkommen ist.

[6] [...] Welicher des handwercks nit enist und aber in den tag [c.-à-d. une réunion] und zuo dem hantweck empfangen wirt, das der dem richter [c.-à-d. au roi] des ersten geben sol ein paar swartzer hentschouch und ein totzent wyßer hoßnestlen, dem schulthessen und dem weibel jettwederm ein paar hentschouch, alles an geverd.

[8] Wurd es sich in deheinem zit allso machen, das iro richter von sich selbs wegen reisen wurd und hilff bedoerffte, denne sol imm das hantwerck ze hilff kommen, ze roß und ze fuoß, dry vierze-

17 Voir Sittler, Les associations (voir n. 3), p. 39-41; Schubert, Fahrendes Volk (voir n. 8), p. 138-140; et pour la Suisse: DUBLER, Handwerk (voir n. 3), p. 86-95; Rudolf WiSSELL, Des alten Handwerks Recht und Gewohnheit II, éd. par Ernst SCHRÄPLER, vol. 7, Berlin ${ }^{2} 1974$, p. $82-123$.

18 SitTLER, Les associations (voir n. 3), p. 40.

19 DUBLER, Handwerk (voir n. 3), p. 88. 
chen tag helffen reyßen, mit dem underscheide, als hienach stat, mit nammen: die ersten vierzechen tag in des hantwecks eigenen costen und zerunge; aber die andern vierzechen tag sol inen der herre costen und zerung geben und keinen sold, aber die dritten vierzechen tag sol inen der herre kost, zerung und sold geben, ze roß und ze fuoß, in der summ, als ze Bern sitt und gewohnlich ist.

[9] Fúrer ist ouch des hantwercks recht: waere, das deheiner, der des tages ist, umb erlich sach gefangen wurd, und das für den herren kumpt, der sol denn haben ein guot pferdt, damit riten und werben und sin allerbest tuon in sinen eigenen costen, untz das der gevangen gelediget wirt. Waere aber sach, das er sin pferit in soelichem dienst abritte und allso unnitz [!] wurd, denn sol im das hantwerck ein anderes, besseres und nit swechers kouffen; und darnach aber sin bestes tuon in des hantwercks costen und zerunge, an alle geverde ${ }^{20}$.

Après la fusion des royaumes de Zurich et de Lucerne, les chaudronniers de ce grand royaume se réunissaient annuellement à Baden, ville préférée par la Diète suisse, devenue un centre politique et administratif. Or, malgré cet avantage, l'intention du roi zurichois de créer un seul royaume suisse échoua. Les rois des chaudronniers suisses, confirmés par leurs villes, étaient en effet des personnages puissants et influents. Dans les années 1480, d'illustres noms apparaissent, comme ceux du noble Adrian de Bubenberg de Berne, fils du vainqueur de la bataille de Morat, ou encore celui du bourgmestre de Zurich, Hans Waldmann. Mais, en 1488, la Diète, lassée de devoir régler les conflits permanents entre les chaudronniers zurichois et lucernois, abolit ce royaume afin de préserver la paix entre les membres au sein de la Confédération ${ }^{21}$.

Nos deux métiers avaient des problèmes communs: les ménétriers, méprisés par l'Église, dispersés dans tout le pays et apparentés à des groupes mobiles en marge de la >bonne sociétéく, avaient besoin d'un protecteur puissant. Leur statut social peu élevé et l'hostilité de l'Église à leur égard les rendaient spécialement vulnérables. Les chaudronniers, dispersés dans les villes et à la campagne, parcouraient leur royaume pour vendre leurs produits. Comme toute corporation, ils s'efforçaient de se défendre contre la concurrence, surtout celle des chaudronniers ambulants. Infâmes et considérés comme valides mendicantes, voire comme espions et incendiaires, les chaudronniers étrangers ambulants furent la cible de mesures draconiennes prises par la Diète ellemême, qui, à partir des années 1480 , leur interdit le territoire suisse ${ }^{22}$.

En dehors des associations de ménétriers ou de chaudronniers, les fêtes des corporations servaient de modèle aux compagnons. Comme tout autre groupe de jeunes gens,

${ }_{20}$ Die Rechtsquellen des Kantons Bern, Erster Teil: Stadtrechte. Achter Band: Das Stadtrecht von Bern, 1. Hälfte: Wirtschaftsrecht, éd. par Hermann RENNEFAHRT, Aarau 1966, p. 456-460: L'association nomme un roi, puis, en même temps, un Schultheiss et un Weibel. Le roi, appellé »seigneur« et »juge«, est présent lors de l'assemblée annuelle. Voir aussi les statuts de Ratsamhausen, SITTLER, Les associations (voir n. 3), p. 40.

${ }_{21}$ En juin 1489, une délégation de chaudronniers venue à Baden chercha à rétablir le royaume, mais les autorités des Confédérés refusèrent leur demande, DuBLER, Handwerk (voir n. 3), p. 92-94.

22 Oliver LANDOlT, »... ich acht, das kaum ein ort sei, do die armen mehr not liden dann im Schwitzer land«. Zur Ausgrenzung der mobilen Armut in der spätmittelalterlichen Eidgenossenschaft, dans: Hans-Jörg GILOMEN, Sébastien GuEX, Brigitte STUDER (dir.), Von der Barmherzigkeit zur Sozialversicherung. Umbrüche und Kontinuitäten vom Spätmittelalter bis zum 20. Jahrhundert, Zurich 2002, p. 127-138. 
les compagnons de métiers avaient leurs fêtes et leurs rituels, en particulier l'élection d'un roi, le jour de la Saint-Nicolas ${ }^{23}$. Il est intéressant de constater que le conseil de la ville de Colmar, en 1421, interdit aux jeunes de la corporation des vignerons et des autres travailleurs de la terre de nommer ces rois avant Noël. La durée du royaume est alors réduite au seul jour des Rois et les activités du roi et de son entourage sont restreintes à la Zunftstube, où il demeure plus facile d'exercer un contrôle sur les jeunes:

Anno 1421 hat der rat und zunftmeistere erkannt, das der ackerlute, der reblute noch keine andere knaben noch knechte keine kunige vor wienachten me haben noch machen sollen deheins wegs, welliche zunft ouch uff den zwolften obent kunige machen wellent, die mogent in uff ire stuben haben und ime do zucht bieten und schencken, su sollent aber nut mit imme umb noch afterwegen riten noch gon in kunigswise, doch mogen su wol ze nacht von den stuben untz heim mit inen gon ungeverlich ${ }^{24}$.

Les autres villes de la vallée du Rhin cherchent de la même manière à discipliner les jeunes et leurs fêtes.

Il y avait donc deux concepts de royauté connus et répandus dans cette région, l'un territorial et politique à la fois, défini par des fleuves et des montagnes qui délimitaient le champ d'action du roi, protecteur de groupes mobiles à l'intérieur de cette aire. L'autre, purement festif, s'inscrivait dans la période des fêtes de Noël. Mais, ce n'est qu'au cours de $\mathrm{XVI}^{\mathrm{e}}$ siècle que le concept de royaume changea son ancienne signification pour devenir synonyme de banquet ${ }^{25}$. Il me semble évident que l'exemple de

23 Robert Muchembled, Die Jugend und die Volkskultur im 15. Jahrhundert. Flandern und Artois, dans: Peter DINZELBACHER, Hans-Dieter MÜCK (dir.), Volkskultur des europäischen Mittelalters, Stuttgart 1987, p. 35-58; Norbert SCHINDLER, Die Hüter der Unordnung. Rituale der Jugendkultur in der frühen Neuzeit, dans: Giovanni LEVI, Jean-Claude SchMiTT (dir.), Geschichte der Jugend. Von der Antike bis zum Absolutismus, vol. 1, Francfort/M. 1996, p. 319-382; Ilaria TADDEI, Fête, jeunesse et pouvoirs. L'abbaye des Nobles-Enfants de Lausanne, Lausanne 1991; Nikolaus GRASS, Royaumes et abbayes de la jeunesse - »Königreiche« und »Abteien« der Jugend. Zum Knaben und Burschenschaftsbrauchtum in Frankreich, in der Schweiz, in Deutschland und in Österreich, dans: Louis C. MORSAK, Markus ESCHER (dir.), Festschrift für Louis Carlen zum 60. Geburtstag, Zurich 1989, p. 411-459; Natalie Zemon DAVIS, The Reasons of Misrule. Youth Groups and Charivari in Sixteenth Century France, dans: Past and Present 50 (1971), p. 41-75; Albert LuTZ, Jünglings- und Gesellenverbände im alten Zürich und im alten Winterthur, Affoltern/A. 1957.

24 Franz J. Mone, Volkssitten und Gebräuche, dans: Zeitschrift für die Geschichte des Oberrheins 20 (1867), p. 78. Cf. aussi Lucien SitTLER, Les mouvements sociaux à Colmar du $\mathrm{XIV}^{\mathrm{e}}$ au XV $\mathrm{XV}^{\mathrm{e}}$ siècle, dans: Revue d'Alsace 95 (1956), p. 129-145, ici p. 135: als ettliche zunffte doher gewonheit gehept hant, das si herren kunige und Epte oder anders desglichen machtent und erbern luten ir kint und knecht jung oder alt viengent. L'interdiction fut répétée en 1436.

25 SEIDENSPINNER, Das »Königreich« (voir n. 4), p. 264, distingue deux phases: dans la première, le royaume sert aux groupes mobiles, voire marginaux, à s'intégrer dans la société et à y participer; dans la deuxième, le royaume devient un haut lieu de la sociabilité. Sur le changement de la signification du mot Königreich, voir Johann et Jacob GRIMM (dir.), Deutsches Wörterbuch, t. 10, Leipzig 1877 (réimpr. Munich 1984), p. 1609-1710; Fritz STAUB et al. (dir.), Schweizerisches Idiotikon. Wörterbuch der schweizerdeutschen Sprache, t. 3, Frauenfeld 1895, p. 159. Dans les régions viticoles, comme à Fribourg en Brisgovie, les étudiants nommaient des rois du vin. Les royaumes du vin liés au carnaval furent interdits par le conseil de ville en 1538, cf. GRASS, Royaumes (voir n. 23), p. 428-432. Selon le protocole de »visitation« de 1549, les 
l'Alsace confirme la coexistence de ces deux concepts, au moins jusqu'à la fin du $\mathrm{XV}^{\mathrm{e}}$ siècle. Les rois (et les abbés) élus par les jeunes des corporations semblaient avoir irrité les autorités de telle manière qu'elles cherchèrent à limiter leur action par des ordonnances. Quant aux royaumes des compagnons, on peut supposer qu'ils s'inspirèrent de ces organisations de jeunesse, les Knabenschaften ${ }^{26}$.

\section{LES ROIS DES COMPAGNONS}

En 1407, le conseil de la ville de Sélestat, en Alsace, fait arrêter les compagnons cordonniers Lindenzwig, venant de Mulhouse, et Rúdin Ams, de Zurich. Les deux hommes venaient de proclamer deux choses considérées comme dangereuses par les autorités de Sélestat. D'une part, les compagnons cordonniers avaient, en 1407, choisi le chevalier Werner, burgrave et bailli d'Alsace, comme seigneur et protecteur de leur association interrégionale, qui s'étendait de Rheinfelden (petite ville suisse des bords du Rhin) à Strasbourg. D'autre part, ils avaient demandé que tout compagnon cordonnier intègre désormais leur organisation. Les deux porte-parole, munis d'une lettre du burgrave, avaient parcouru la région pour inciter leurs collègues à se joindre à leur association et à participer à l'assemblée générale qui devait avoir lieu à Rouffach en octobre 1407 et qui serait présidée par Werner en personne:

Ich, Lindenzwig von Múlnhusen, und ich, Rúdin Ams von Zúrich, die schuomacherknechte, vergehent, als wir zuo Sletzstat gefangen gewesen sind, davon als wir wurbent, das alle schuomacherknechte in allem disem lande hern Wernher Burggraven ritter zuo eim rechten herren haben soltend und ouch des sin offenn brieff umbtruogent, und was das ein solich uffsatz, davon dem gemeinen lande großer breste moechte ufferstanden $\sin ^{27}$.

En effet, de 1407 à 1408, les compagnons cordonniers étaient en train de former une vaste alliance interrégionale, très contestée par leurs maîtres comme par les conseils de villes de la vallée du haut Rhin. Pour confirmer leur indépendance par rapport à leurs maîtres de métier et certaines autorités urbaines, ils avaient opté pour ce modèle. En choisissant un seigneur capable de les protéger et de défendre leurs intérêts, les compagnons avaient créé un précédent, jugé extrêmement dangereux par les villes. Dans sa lettre, le burgrave Werner avait proclamé:

Das die schuomacher knechte gemeinlich in obern und niedern Eilsasz zwúschent Rinfelden und Strassburg mich einhelleklich erwelt und erkosen habent vúr iren rehten herren, harumbe erkenne ich mich, das ich dieselben schuohemacher knehte, wo und welhen stetten sie danne wo-

autorités constataient Bursales in quadragesima die Veneris bacchanalia, quae solent vocare Regnum celebrasse, cantavisse, clamasse et saltasse saepius ad mediam usque noctem, ibid., p. 430.

26 Les royaumes suisses auraient été influencés par les organisations de jeunesse, cf. REININGHAUS, Die Entstehung (voir n. 5), p. 54.

27 Elsässische Stadtrechte, vol. 1: Schlettstadter Stadtrecht, éd. par Joseph GÉNY, Heidelberg 1902, p. 616 (9 avril 1408). 
nende sind in minen schirm und vúr die minen empfangen habe und wil inen beraten und beholffen $\sin$ zuo rehten, als verre ich kan und mag ${ }^{28}$.

Ayant abandonné le cadre de leur corporation, les compagnons avaient en même temps renversé la hiérarchie traditionnelle et miné l'autorité et la juridiction des villes. De plus, en 1407, sur les deux bords du Rhin, en Alsace et au pays de Bade, le bruit courrait que les compagnons cordonniers avaient l'intention d'organiser un arrêt généralisé du travail et que ce projet serait discuté lors d'une assemblée à Sélestat.

Dans la région du haut Rhin, les compagnons cordonniers avaient adopté le modèle du royaume avec un roi issu de la noblesse. Dans le nord-est de la Suisse, les compagnons dispersés dans les nombreuses petites villes s'étaient organisés de la même manière, mais, à la différence des leurs collègues, avec un roi élu parmi l'un des leurs. Un rare document, datant de 1421, nous présente cette association et son rayon d'action. Cette sentence arbitrale est le résultat de longues discussions menées lors de l'assemblée annuelle des compagnons à Zurich. Confirmée par les autorités zurichoises, elle est signée d'abord par les maîtres et ensuite par les trois fonctionnaires à la tête de l'association: le roi, l'écoutète et l'huissier (König, Schultheiss et Weibel); six autres compagnons sont témoins:

Wir der burgermeister und die raet der statt Zürich tuont kunt menglichem mit disem brief von der stoess und misshellung wegen, so bisher etzwas zites gewesen sint zwueschent den ersamen wisen den zunftmeistern, den meistern, den zúnften und den gesellschaften schuomacher hantwerchs diser nachgeschriben stetten mit nammen Constenz, Überlingen, Schaffhusen, Winterthur, Lucern, Arow, Bremgarten, Baden, Brugg, Keiserstuol und Louffenberg [...] die vorgenanten zunftmeister und meister in nammen als vor und die egenanten Hanns Holdermeyer, kúng, Ulrich Keller, schultheiß, und Hanns Krieg, weybel, ouch in nammen ir selbs und der vorgenanten ir mitgesellen der schuochknechten und für sy mit iren trúwen gelopt und versprochen, [...] das sy das ze beider sit fúr sich und die iren war und staet halten und dem gnoug tuon sullen und wellen aene geverde [...]. Wir haben ouch in disem unserm spruch den vorgenanten schuochknechten uffgesetzet und vorbehept, das sy einen kúng, einen schultheissen und einen weibel und ouch iren meyen haben mugen, ob sy wellen, doch den vorgenanten stetten, dem meistern, den zúnften und gesellschaften schouchmacher hantwerchs der selben stetten und ouch disem unserm spruch gentzlich unschedlich, an alle geverde ${ }^{29}$.

28 Johannes FRITZ, Der Ausstand der oberrheinischen Schuhmachergesellen im Jahr 1407, dans: Zeitschrift für die Geschichte des Oberrheins 45 (1891), p. 132-140, ici p. 134.

29 Friedrich Emil Welti (éd.), Die Urkunden des Stadtarchivs zu Baden im Aargau, Berne 1896, p. 344-345, $\mathrm{n}^{\circ} 387$ (9 juillet 1421), les corporations des villes de Constance, Überlingen, Schaffhouse, Winterthur, Lucerne, Aarau, Bremgarten, Baden, Brugg et Kayserstuhl avaient délégué des maîtres, l'autre parti, Johans Holdermeyer von Zúrich kúng, Ulrich Keller von Winterthur, Schultheß, und Hans Krieg von Bremgarten, weibel der vorgenanten schúchknechten, avait envoyé ses témoins venant de Rapperswil, Bremgarten, Schaffhouse, Lucerne, Brugg, Baden. Cette ville de bains très fréquentée et célébrée par l'humaniste italien Poggio, était un centre commercial et administratif important de la Confédération suisse, qui attirait, notamment, beaucoup de compagnons étrangers, voir Willi GAUTSCHI, Beitrag zur Sozialgeschichte der Stadt Baden im 15. Jahrhundert, dans: Argovia 72 (1960), p. 134-153; Hektor AmmanN, Die Stadt Baden in der mittelalterlichen Wirtschaft, dans: Argovia 63 (1951), p. $217-321$. 
Cette sentence arbitrale règle certains différends non spécifiés. Elle insiste cependant sur le problème de la juridiction et oblige les deux parties opposées à se soumettre au tribunal de la corporation ou de la ville où le conflit a lieu. De même, les deux parties renonceront à faire appel à d'autres tribunaux. Les compagnons, à leur tour, régleront leurs différends internes de la même manière. Selon le compromis signé par les opposants, les maîtres confirment aux compagnons le droit de s'associer et de nommer le roi et les deux autres fonctionnaires ainsi que de se rassembler une fois par an comme auparavant, ils sont prêts à garantir ces droits sous réserve que l'on ne nuise pas aux villes mentionnées ci-dessus.

Rappelons que le document est signé par le roi des compagnons forgerons, en 1412. Nous y retrouvons à peu près la même situation et les mêmes arrangements. Les maîtres respectent l'association et la fonction du roi. Le roi, à son tour, promet d'empêcher le moindre acte qui pourrait nuire à la corporation et à ses maîtres:

Es ist ze wússent umb die stoess und misshellung, so der schmid knechten kúng und gemein schmid knecht bis her gehept hand mit den meistern und gemeiner zunft schmid antwerks ze Zúrich, wie die stoess sich hieschent umb all vergangen sachen bis uff disen húttigen tag, daz wir $d a$ ze beider sit mit guoter vorbetrachtung und nach raet einer frúntschaft und einhelligi mit einander úber komen sind, als hie nach geschriebn staet: Des ersten ist berett, daz ich obgen(ant) kúng noch deheiner min nach kom noch alle die knecht unsers antwerks, so ietz sind oder hie nach werdent, den ob gen[anten] meistern ze Zürich noch ir nach komen deheinen knecht hinnenthin eweklich niemer me verbieten sond, inen ze werken [...]. Wer och, daz hinnenthin dehein knecht mit sim meister icht ze schaffen hett, daz si mit ein ander stoess gewunnent, der selben stoessen sond beid teil, meister und knecht, fúr die zunftmeister schmid antwerks komen und fúr die sechs oder zwelf. Múgent si denn vor dien bericht werden, daz ist wol und guot; bschaech daz nit, so sont aber beid teil der sach fúr den schulth[eiss] komen und da waer und staet halten ze beiden siten, waz inen da erteilt und ussgesprochen wirt, es tuo wol oder we, wie dik daz ze schulden kunt ${ }^{30}$.

Le premier paragraphe aborde le problème du boycottage. Le roi des compagnons s'engage, pour lui-même et pour ses successeurs, à renoncer à cette mesure aussi efficace que redoutée par les corporations et les conseils de villes. Les paragraphes suivants prescrivent la procédure en cas de conflit. Selon cet accord, les deux parties auront recours au tribunal de la corporation, qui servira de première instance; ils auront le droit de faire appel au tribunal communal en dernière instance, dont le jugement devra être accepté par les deux parties. Par cet accord, les maîtres et les compagnons promettent de respecter la juridiction communale et de renoncer à faire appel aux tribunaux étrangers.

Dans un troisième document, daté de 1424 , le ton change complètement. La situation est la suivante: deux compagnons avaient, malgré l'accord conclu en 1421, osé boycotter les maîtres cordonniers dans la ville de Baden. Selon quoi, les autorités de la ville les avaient accusés d'avoir rompu leur serment. Cette fois, la petite ville de Rheinfelden, où les deux coupables avaient été arrêtés, arbitra le conflit entre les maîtres, décidés à punir sévèrement les deux réfractaires, et les compagnons cordonniers

30 SCHNYDER (éd.), Quellen zur Zürcher Zunftgeschichte (voir n. 1), vol. 1, n 58, p. 68. 
venus pour implorer leur grâce ${ }^{31}$. La sentence arbitrale ne se borne pas à punir les deux coupables, elle met fin aux fonctions du roi, de l'écoutète et de l'huissier, ainsi qu'à l'assemblée annuelle (Maien). De plus, elle interdit explicitement aux compagnons cordonniers d'exercer leur juridiction interne. Désormais, chaque délit sera réglé par les tribunaux de la corporation ou de la ville et, selon la nouvelle sentence arbitrale, tout réfractaire ayant violé le serment sera puni de mort:

Wir der schultheß und der rat ze Rinvelden tuont kunt aller menglicher mit diesem briefe: als Marti Stettbach von Gisingen, der schuochknecht, dem meistern schuochmacher hantwercks ze Baden knechte verbotten und Guntfried Swartz der schouchknecht von Brúnlingen darumbe botte gewesen were und den verbietbrieff da selbs hingetragen hette [...] darumbe unser lieben guoten frúnde, schultheiss und rate ze Baden, uns ernstlichen tatten bitten, die egenannten Marti Stettbach und Guntfried Swarzen inen uf recht ze haltent, das wir durch ir bette willen tatten und si in leiten. [...] Es soellen och die spruchbrieff, als die obgenannten unser frunde von Zúrich gesprochen und versigelt gegeben hand, by allen iren kreften, puncten und artickeln nach irem innehalten beliben, ußgenommen umbe den kúng, schultheissen, weibel und meyen ze machende, das sollent sy hynnethin nit tuon, als vor statt 32 .

Ces trois documents nous informent implicitement sur les fonctions du roi des compagnons. Il semble évident que les rois (et les deux autres fonctionnaires) servaient à structurer ces associations, qui englobaient de nombreuses petites villes de la Confédération suisse et de l'Allemagne. En 1412 et en 1421, les rois des compagnons forgerons et cordonniers, porte-parole de leurs associations respectives, semblent avoir été des partenaires respectés, capables de calmer les tensions au sein de leur groupe. Les documents nous confirment qu'ils rédigeaient et signaient des accords en commun avec les maîtres et que les rapports étaient plutôt détendus, par comparaison avec la situation dans villes du haut Rhin. Selon les rares documents conservés, les rois (et les autres fonctionnaires) parvenaient à négocier avec les maîtres et les conseils de villes, tout en défendant leurs intérêts. En revanche, les rois étaient supposés faire respecter l'accord conclu entre les deux parties et surtout empêcher leurs collègues de recourir au boycottage. Les maîtres espéraient donc discipliner les compagnons par ces intermédiaires, ce qui s'avéra une mission impossible. Soit les rois, ayant juré de s'abstenir de boycotter et se soumettant aux tribunaux de la corporation ou de la ville, n'arrivaient pas à imposer leur autorité aux compagnons refusant de leur obéir, soit les associations interrégionales présumaient de leurs forces, en regard des succès remportés par leurs collègues de la vallée du haut Rhin. En tout cas, les rois qui étaient supposés coopérer avec les maîtres et œuvrant au maintien de l'ordre devaient répondre des infractions.

31 Welti (éd.), Die Urkunden des Stadtarchivs zu Baden (voir n. 29), p. 370-273, n. 416 (7 août 1424): Bâle, Zurich, Schaffhouse, Waldshut, Kayserstuhl, Aarau, Winterthur, Bremgarten, Brugg et Säckingen avaient délégué des maîtres et des magistrats afin de punir les deux coupables; en revanche, les 33 compagnons qui sont intervenus en faveur des inculpés venaient des villes du royaume, de Bâle et même de Ravensburg, Augsbourg, Fribourg/Br. et Ingolstadt.

32 Ibid. p. 372: Quoique l'accord conclu en 1421 soit confirmé, les fonctionnaires et l'assemblée seront abolis. Voir aussi GAUTSCHI, Beitrag (voir n. 29), p. 144-147. 
Avant d'analyser les changements qui menèrent à l'interdiction des associations interrégionales et des rois des compagnons, il faut se rappeler que les trois documents de 1412,1421 et 1424 que nous venons d'analyser s'inscrivent dans ce qu'il convient d'appeler »le mouvement des compagnons de métiers«. Au début du $\mathrm{XV}^{\mathrm{e}}$ siècle, les villes de la vallée du haut Rhin furent le théâtre de luttes violentes opposant les compagnons et leurs maîtres. L'impact fut tel que les villes du haut Rhin et celles du haut Rhin moyen (comme Mayence) décidèrent de coordonner leurs pouvoirs de coercition à l'égard des compagnons ${ }^{33}$.

Les régions avoisinantes ne furent pas épargnées par ce conflit. Les compagnons forgerons et cordonniers habitant les petites villes d'Argovie, de Lucerne et des deux bords du Rhin étaient aussi membres d'associations interrégionales dans lesquelles les petites villes servaient de lieux d'assemblée. En plus, les compagnons avaient créé des réseaux de communication qui fonctionnaient aussi bien que ceux de leurs maîtres. Ces réseaux parallèles comprenaient les petites villes et villages du rroyaumeく des compagnons ainsi que certains centres urbains où les compagnons avaient créé des organisations locales ${ }^{34}$.

\section{LA FIN DES ROIS DES COMPAGNONS}

Le modèle d'organisation interrégionale conduit par un roi était contesté depuis longtemps, nous l'avons vu. Dans une lettre de 1420 envoyée aux autorités de Zurich, les maîtres forgerons de la ville de Rottweil demandèrent au conseil de ville de Zurich de suivre leur exemple et d'abolir le royaume des compagnons forgerons, responsable, selon eux, de troubles. Pour convaincre les villes de la Confédération suisse, ils brandirent la menace d'une conjuration dangereuse menaçant les villes et la campagne et terrorisant maitres et compagnons ${ }^{35}$. Malheureusement, nous ne connaissons pas les réactions provoquées par l'intervention de Rottweil. Ce n'est qu'en 1424 que les auto-

33 Déjà en 1387, les maîtres forgerons de Francfort/M., Mayence, Worms, Speyer et d'autres villes avaient conclu une alliance contre les compagnons devenus trop indépendants. Il faut bien se rendre compte que les compagnons travaillant dans les villes du haut Rhin avaient des rapports étroits avec leurs collègues du moyen Rhin. En 1399, les villes du haut Rhin, le bailli d'Alsace et plusieurs petits seigneurs prirent des mesures contre les compagnons cordonniers dont les activités associatives provoquèrent de vives réactions de la part des villes du haut Rhin et du moyen Rhin en 1407/1408, de même que les activités des compagnons tailleurs à Constance en 1410 alarmèrent les villes du haut Rhin, la correspondance entre les villes en est témoin, voir SCHULZ, Handwerksgesellen (voir n. 5), p. 68-81.

34 Reininghaus, Die Entstehung (voir n. 5), emploie le terme Gesellengilden comprenant des aspects sociaux, économiques, politiques, confraternels.

35 Ibid., p. 87: geloppte gesellschaft; HEGI, Zunft (voir n. 1), p. 128. De même, les villes situées le long du Rhin, de Bâle à Bingen, s'engagèrent à prendre des mesures contre les associations de compagnons, en demandant aux compagnons nouveaux venus de jurer dans un délai de trois jours de ne pas devenir membres d'une association, voir SCHULZ, Handwerksgesellen (voir n. 5), p. 72-73. 
rités des villes suisses inclinèrent définitivement et de manière négative leur politique vis-à-vis des rois et des royaumes des compagnons.

Il y eut surtout trois gravamina majeurs qui poussèrent alors les villes à supprimer ce genre d'associations:

Primo, le modèle du royaume et du seigneur protecteur posait de sérieux problèmes à l'autorité et à la juridiction des villes, qui se défendaient énergiquement contre toute tentative pouvant les concurrencer ou, pis encore, les miner. Rappelons qu'en 1407, pour confirmer leur indépendance par rapport aux corporations et aux tribunaux communaux, les compagnons cordonniers avaient choisi comme patron le bailli d'Alsace. En effet, le burgrave Werner, prenant au sérieux ses obligations, s'efforça de libérer les deux cordonniers emprisonnés à Sélestat. Mais, par sa lettre adressée aux autorités de cette ville, il versa de l'huile sur le feu en déclarant que l'ordonnance que les compagnons étaient en train de rédiger ne regardait personne, même pas leurs maîtres, et que c'était à lui seul de juger et de punir en conséquence les compagnons cordonniers ${ }^{36}$. Dans son esprit, il s'agissait d'un acte tout à fait légitime de la part des compagnons. Il est évident que son argument confirmait les pires craintes des corporations et des conseils de villes. Par conséquent, ils cherchèrent à empêcher à tout prix que d'autres compagnons ne suivent l'exemple des cordonniers.

Lorsque les maîtres et les compagnons potiers de la région allant de Ravensburg à Strasbourg, en 1435, choisirent le chevalier, magistrat et diplomate bâlois, Henman Offenburg comme seigneur et chef, les villes et même ses pairs bâlois, craignant le précédent créé par les cordonniers en 1407, s'inquiétèrent. Offenburg, en bon diplomate, arriva à calmer les esprits en niant strictement les parallèles entre l'initiative des potiers et celle des compagnons cordonniers arrogants vis-à-vis de leurs maîtres ${ }^{37}$. Selon les statuts, les potiers du »pays bas« avaient leur assemblée annuelle le jour de la Pentecôte à Brisach, ceux du »pays haut« à la Saint-Jacques à Ravensburg. Au $\mathrm{XV}^{\mathrm{e}}$ siècle, ces assemblées duraient jusqu'à deux semaines ${ }^{38}$. En effet, les potiers, dispersés dans les villes et les villages, ne posaient aucun problème, mais la structure de l'association interrégionale en elle-même, avec à sa tête un seigneur unique qui

36 FRITZ, Ausstand (voir n. 28), p. 134.

37 Selon Offenburg, il ne serait point raisonnable de comparer les deux cas, puisque les compagnons cordonniers à la différence des potiers, ayant perverti l'ordre, auraient den Burggrafen fur iren herren uffgeworfen glicher wis als ein freyer man sich einen herren eignete. Il insiste sur le fait qu'il y avait, depuis 1419, une association de potiers en Alsace, voir ScHULZ, Handwerksgesellen (voir n. 5), p. 82-86; Elsanne GILOMEN-SCHENKEL, Henman Offenburg (13791459). Ein Basler Diplomat im Dienste der Stadt, des Konzils und des Reichs, Bâle 1975, p. 106-110. Peu d'informations sur la structure de l'association dans Susanne EuLES, »Der hafner gesellen lobliche bruderschaft«. Organisation der Hafnerbruderschaft und Erzeugnisse der Hafner des 15. bis 18. Jahrhunderts im Elsaß, Sundgau und Breisgau, Francfort/M. 1991.

38 Ibid. p. 43. Malheureusement, il n'y a pas d'informations, ni sur la structure ni sur ces assemblées, au moins pour le $X^{\mathrm{e}}$ siècle. Selon la description de l'assemblée à Brisach en 1604, elle commença par la séance du tribunal qui collectionnait les amendes, puis on accueillit les nouveaux membres avant le compte rendu du comité, la partie officielle se termina par une messe. Selon Eules, une danse et un cortège s'ensuivirent, mais elle n'évoque pas de repas commun, partie importante des fêtes des confréries, ibid., p. 44-45. 
défendait les membres et qui exerçait la justice, remettait en question l'autorité des villes.

Secundo. Chacune des assemblées inquiétait les conseils des villes. La nouvelle d'une assemblée organisée par les compagnons provoquait des rumeurs, des craintes et de violentes réactions de la part des villes. Vu les activités des compagnons cordonniers, l'assemblée qui devait se tenir à Rouffach, à l'hiver 1407, fut considérée comme un très grand danger, et, par conséquent, les villes de Bâle et Mayence cherchèrent à l'empêcher. La vive correspondance entre les villes de la vallée du haut Rhin, et entre Bâle, Mayence et d'autres villes du haut Rhin moyen reflète cette crainte. D'une part, les villes s'attendaient à voir se réunir un très grand nombre de participants (Bâle, dans sa lettre, parle même de quatre mille compagnons) et, d'autre part, les autorités craignaient surtout que d'autres compagnons, notamment les compagnons tailleurs, très actifs aussi, ne suivent l'exemple donné par les cordonniers. Dans leurs correspondances, elles formulent leurs craintes ainsi: daz snider und ander antwerkknechte dasselb oder desglich ouch underston und herren úber ir meister wurden ${ }^{39}$.

En 1408, les compagnons de Strasbourg et ceux de Haguenau invitent leurs collègues à se rassembler dans la ville d'Haguenau. Par une lettre, les organisateurs avaient mentionné leur intention de régler, une fois pour toutes, les rapports (déjà tendus) entre les compagnons et leurs maîtres. Les villes, apprenant que les compagnons de trente et une petites villes avaient déjà accepté l'invitation et craignant le précédent des cordonniers, s'apprêtèrent à réagir ${ }^{40}$.

Bien que les deux assemblées, celle de Rouffach et celle de Haguenau, n'aient pas eu lieu, les maîtres, se sentant menacés par les compagnons, se réunirent en 1408 à Sélestat pour discuter des mesures à mettre en œuvre. De Bâle à Mayence, on parla alors d'abolir les associations locales et interrégionales des compagnons et même de supprimer leurs confréries ${ }^{41}$.

Tertio. L'autre point délicat que nous devons aborder est celui de la juridiction exercée par les associations urbaines et interrégionales des compagnons de métiers. Tant qu'ils se bornèrent à sanctionner leurs collègues ayant violé les règles du métier et blessé l'honneur de la corporation ou bien de régler les querelles internes, leur juridiction ne fut pas contestée. Dans ce cadre bien délimité, cette justice servait à maintenir la paix et à soulager les tribunaux de la corporation et de la ville ${ }^{42}$. Mais, bientôt, les compagnons s'efforcèrent d'élargir leur champ d'action à l'intérieur comme à l'extérieur de leur groupe. Ils imposèrent leur juridiction, non seulement à leurs pairs, ce qui fut toléré dans une certaine mesure, mais aussi à leurs maîtres. L'usurpation de la sphère juridique réservée aux maîtres et les moyens employés pour faire céder leurs opposants provoquèrent l'exaspération des maitres de la profession. Le moyen de coercition le plus effectif et le plus redouté par les corporations et les villes était le

39 Fritz, Ausstand (voir n. 28), p. 133; SitTLER, Les mouvements (voir n. 24), p. 132; SCHUlZ, Handwerksgesellen (voir n. 5), p. 73-75. Sur les conflits précédents, voir SiTTLER, Les mouvements (voir n. 24), p. 131-132.

40 FriTZ, Ausstand (voir n. 28), p. 135, avec la liste des 31 villes alsaciennes.

41 Sur ces discussions, voir SchUlz, Handwerksgesellen (voir n. 5), p. 74-75.

42 ReIninghaus, Die Entstehung (voir n. 5), p. 94. 
boycottage, soit d'un atelier, soit de tout le métier. Promulgué dans tout le pays, le boycottage privait l'atelier en question de compagnons, et ceux qui osaient travailler dans l'atelier frappé d'interdit risquaient d'être à leur tour boycottés par la profession $^{43}$. De cette manière, le maître, ou sa corporation, était contraint de céder face aux compagnons et de se réconcilier avec leur association. Afin de se protéger contre ces mesures, les maîtres et les conseils de villes, comme à Mayence et à Strasbourg, coordonnèrent leurs actions jusqu'à la rédaction de l'ordonnance définitive qui réglementait le statut des compagnons et leurs devoirs par rapport aux maîtres, en réduisant nettement leur prérogatives. Cette ordonnance, nommée »Rheinische Knechtsordnung«, fut promulguée dans la deuxième moitié du $\mathrm{XV}^{\mathrm{e}}$ siècle ${ }^{44}$.

Dans les trois documents que nous venons d'analyser, ce problème du boycottage joue un rôle crucial. À travers eux, en 1412 et en 1421, nous voyons le roi des compagnons forgerons et celui des compagnons cordonniers jurer de s'abstenir de boycotter au nom de leur association. En 1412, le roi des compagnons forgerons arrive encore à faire amnistier un compagnon qui avait promulgué le boycottage de son maître ${ }^{45}$. Mais ces rois, intermédiaires entre les compagnons et les maîtres, s'avéraient incapables d'imposer cette restriction qui privait les compagnons de leur arme la plus efficace, comme le montre clairement le cas des deux compagnons réfractaires mentionnés cidessus, qui servirent de prétexte pour supprimer les associations interrégionales des compagnons.

Il y avait, bien sûr, bien d'autres points litigieux entre maîtres et compagnons, comme par exemple les repas et le temps libre accordés à ces derniers, mais également les rituels par lesquels les compagnons accueillaient leurs nouveaux collègues. Ces rituels furent âprement discutés, car ils avaient lieu pendant les heures de travail. Contestés par les maîtres et défendus par les compagnons, ils servaient à établir des rapports étroits entre l'association et ses nouveaux membres, des moments essentiels pour confirmer l'identité du groupe.

\section{CONCLUSION}

Au cours du bas Moyen Âge, les compagnons de métiers adoptèrent différents modèles d'associations connues dans la région du haut Rhin et en Suisse. En Alsace, en Brisgovie et dans le nord-ouest de la Confédération suisse, ils créèrent eux-mêmes des royaumes fondés sur le modèle de groupes ambulants, dont les exemples les plus connus sont les ménétriers alsaciens et les chaudronniers. Présidées par un roi (chez les compagnons forgerons) ou par un comité de trois représentants (roi, écoutète, huissier chez les cordon-

43 Plutôt que de risquer de se faire exclure de l'association, les compagnons préféraient suivre le boycottage et se faire punir par la corporation et le conseil de ville, voir SCHULZ, Handwerksgesellen (voir n. 5), p. 71.

44 Ibid., p. 81-98; Wilfried ReInINGHAus, Die Straßburger »Knechteordnung« von 1436. Ihre Entstehung und ihre Bedeutung für die Geschichte der Gesellengilden am Oberrhein, dans: Zeitschrift für die Geschichte des Oberrheins 87 (1978), p. 131-143.

45 SCHNYDER (éd.), Quellen zur Zürcher Zunftgeschichte (voir n. 1), vol. 1, p. 68, n. 58. 
niers), ces associations interrégionales se rassemblaient une fois par an (Maien), de préférence à Zurich ou dans les petites villes situées en Alsace, en Argovie ou en Brisgovie.

À la différence des ménétriers (Pfeiferkönigreich) et des chaudronniers, dont le royaume était délimité par des frontières géographiques naturelles (des montagnes, des fleuves ou de grandes forêts), les rois des compagnons n'avaient pas de royaume au sens topographique. Leur aire se définissait selon les lieux d'origine de leurs représentants, elle s'étendait de la Forêt-Noire au lac de Constance jusqu'en Argovie.

Placés à la tête d'une association interrégionale dont les membres étaient dispersés en petit nombre dans un réseau de petites villes, les rois servaient d'intermédiaires d'une part entre les compagnons eux-mêmes et d'autre part entre les compagnons et les maîtres ou les autorités urbaines. En tant que porte-parole des compagnons et partenaires des maîtres, ils exerçaient une certaine autorité, qui leur permit de conclure des accords en leur nom ou au nom de leurs associations. Les documents ont montré qu'ils négociaient directement avec les maîtres d'une corporation ou encore devant leurs tribunaux en défendant leurs prérogatives. Les documents signés par les rois et confirmés par leurs sceaux reflètent leur statut si particulier.

Mais, dès les années 1420, la situation s'aggrave. Ces associations interrégionales et leurs activités devenues illicites provoquèrent la mise par écrit d'ordonnances de la part des villes, comme à Mayence et à Strasbourg. Le problème le plus important était la juridiction usurpée par les compagnons. En jugeant leurs maîtres et surtout en boycottant leurs ateliers et même des villes entières, les compagnons avaient dépassé les frontières de ce que les maîtres et les autorités des villes toléraient jusqu'alors. Donc, au plus fort du conflit, en 1424, en coordonnant leurs moyens de coercition, les autorités décidèrent d'abolir les associations interrégionales des compagnons en brandissant la peine de mort.

On constate que les autorités chargées de préserver la paix entre maitres et compagnons confirmèrent ces associations, leur hiérarchie et le droit de s'assembler jusqu'en 1424. De cette manière, les autorités déléguèrent le contrôle des compagnons dispersés dans cette vaste région, afin de régler leurs affaires internes. Mais il est évident que ces rois, médiateurs en cas de conflits (très fréquents et parfois très violents dans la vallée du haut Rhin), n'arrivaient pas à remplir leur devoir, le plus important et le plus difficile, celui d'empêcher leurs collègues de boycotter leurs maîtres et de les conduire à se soumettre à la juridiction des conseils de villes comme ils l'avaient juré.

Les ordonnances et les documents judiciaires peuvent encore révéler d'autres aspects de la culture des compagnons si nous les approchons selon différentes perspectives: les recherches sur le folklore, qui fut longtemps négligé par les historiens, nous rendent visible l'importante place que les compagnons tenaient dans les fêtes et rituels religieux des villes. Dans ce contexte, nous n'avons évoqué que la fête des Rois, mais les repas festifs et l'apparition publique des rois furent aussi limités par les ordonnances des villes.

Les études sur la criminalité, en revanche, peuvent servir à analyser les activités de ces groupes, considérées comme déviantes et sanctionnées par les autorités urbaines. Elles mettent l'accent sur le comportement de la jeunesse masculine, en observant les activités nocturnes, les tavernes, les provocations et les bagarres entre compagnons de 
différents métiers, les violences et les actes de vengeance dirigés contre les maîtres (très rares cependant).

L'histoire sociale s'est bornée trop souvent à réduire ces jeunes gens à de purs syndicalistes avant l'heure. Bien sûr, cet aspect était important, et il serait erroné de le nier. Mais il faut bien se rendre compte qu'il s'agit de jeunes gens impliqués dans toutes sortes d'activités et, point important, intégrés aussi bien dans les rituels festifs et religieux de leurs villes et villages que dans leurs associations et leurs confréries locales.

L'aspect memoria ne doit pas être non plus négligé. Les cierges et les draps mortuaires, très somptueux parfois, reflètent les rivalités existant entre les métiers. Dans ce domaine, la représentation et l'honneur jouent un rôle considérable pour les compagnons et leurs rois.

L'histoire des rois des compagnons est étroitement liée, d'une part, aux royaumes des groupes professionnels ambulants et, d'autre part, aux organisations de jeunesse et à leurs rituels. Mais il s'avère difficile de retrouver des éléments caractéristiques appartenant à la culture des compagnons au niveau de leurs associations interrégionales et de leurs assemblées annuelles, au moins pour le $\mathrm{XV}^{\mathrm{e}}$ siècle. Pourtant, les divers documents analysés nous livrent des indices, et les assemblées tenues par les royaumes des ménétriers, des chaudronniers et des potiers, assez bien connues, nous permettent de dresser une liste d'éléments constitutifs du royaume des métiers: l'accueil des nouveaux membres, le tribunal, le compte rendu des fonctionnaires, la discussion des affaires politiques, ensuite la messe et le repas commun. 